\title{
Pathogenesis of graft-versus-host disease: innate immunity amplifying acute alloimmune responses
}

\author{
Yoshinobu Maeda
}

Received: 18 July 2013/Accepted: 19 July 2013/Published online: 28 August 2013

(C) The Japanese Society of Hematology 2013

\begin{abstract}
In addition to reduced-intensity conditioning, which has expanded the eligibility for hematopoietic cell transplantation (HCT) to older patients, increased availability of alternative donors, including HLA-mismatched unrelated donors, has increased access to allogeneic HCT for more patients. However, acute graft-versus-host disease (GVHD) remains a lethal complication, even in HLAmatched donor-recipient pairs. The pathophysiology of GVHD depends on aspects of adaptive immunity and interactions between donor T-cells and host dendritic cells (DCs). Recent work has revealed that the role of other immune cells and endothelial cells and components of the innate immune response are also important. Tissue damage caused by the conditioning regimen leads to the release of exogenous and endogenous "danger signals". Exogenous danger signals called pathogen-associated molecular patterns and endogenous noninfectious molecules known as damage-associated molecular patterns (DAMPs) are responsible for initiating or amplifying acute GVHD by enhancing DC maturation and alloreactive T-cell responses. A significant association of innate immune receptor polymorphisms with outcomes, including GVHD severity, was observed in patients receiving allogeneic HCT. Understanding of the role of innate immunity in acute GVHD might offer new therapeutic approaches.
\end{abstract}

Keywords Innate immunity - Danger signals .

PAMPs · DAMPs · GVHD

\footnotetext{
Y. Maeda $(\bowtie)$

Department of Hematology and Oncology, Okayama University Graduate School of Medicine, Dentistry, and Pharmaceutical

Sciences, Shikata-cho 2-5-1, Kita-ku,

Okayama, Okayama 700-8558, Japan

e-mail: yosmaeda@md.okayama-u.ac.jp
}

\section{Introduction}

Allogeneic hematopoietic cell transplantation (HCT) is used to treat many hematologic malignancies. However, acute graft-versus-host disease (GVHD) remains the most important complication of allogeneic HCT. GVHD was initially reported by Barnes [1] and Billingham [2] who identified three prerequisites for the development of GVHD: (1) the graft must contain immunologically competent cells; (2) the recipient must be incapable of rejecting the donor cells; and (3) the recipient must express tissue antigens that are not present in the transplant donor. Mature donor T-cells were identified as the fundamental cellular mediators of GVHD and several convergent lines of experimental data have demonstrated that host and donor antigen-presenting cells (APCs), especially dendritic cells (DCs), are critical for the induction of GVHD [3, 4]. In addition to T-cells and DCs, several other cellular subsets, such as B cells, macrophages, $\gamma \delta \mathrm{T}$-cells, NK cells, and NKT cells, are involved in the pathogenesis of GVHD. The past decade has brought impressive advances in our understanding of the role of innate immune responses in the pathogenesis of GVHD. A conditioning regimen that includes total body irradiation (TBI) or chemotherapy damages the host tissues [5]. Injured, stressed, or dying cells release exogenous and endogenous "danger signals" (Fig. 1). This article reviews the importance of the innate immune response activated by danger signals in GVHD.

\section{Triggers that induce GVHD: pathogen-associated molecular patterns (PAMPs)}

After a conditioning regimen, tissue damage in the gastrointestinal (GI) tract allows the transit of bacteria. To 


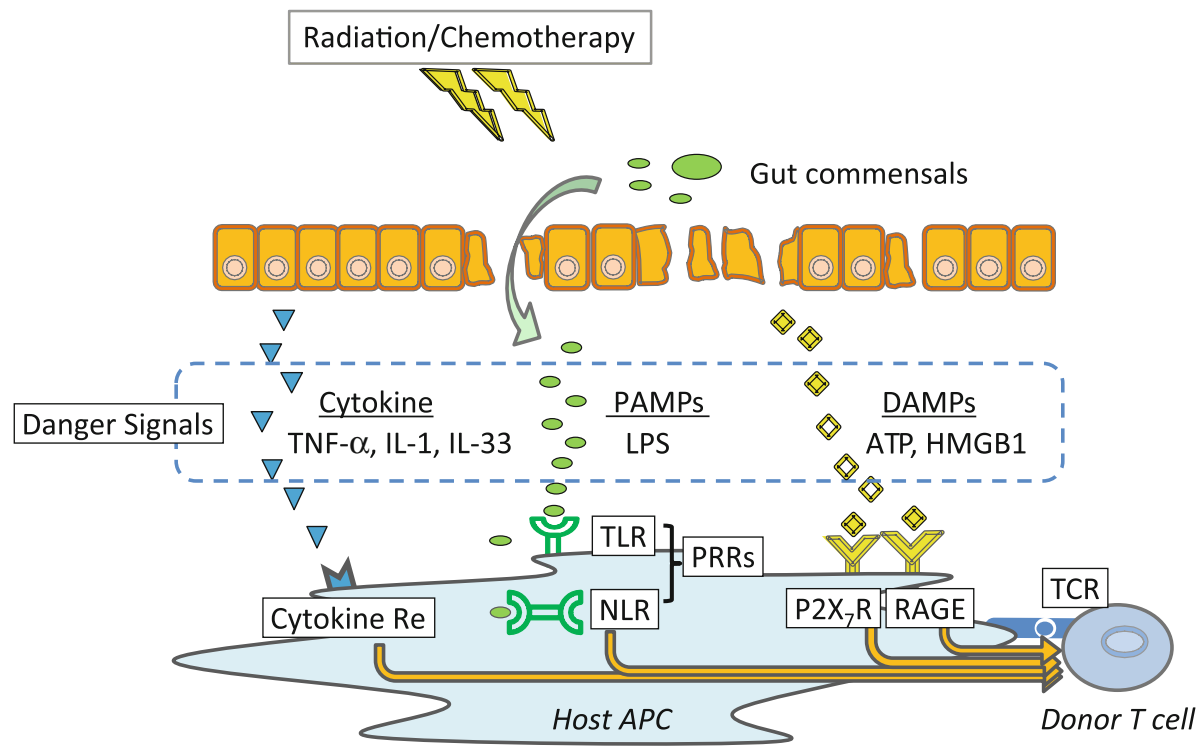

Fig. 1 Importance of innate immune response activated by danger signals in GVHD. The conditioning regimen which includes total body irradiation and/or chemotherapy leads to the damage of host tissues. Damaged cells release "danger signals" including cytokines, exogenous pathogen-associated molecular patterns (PAMPs), and endogenous damage-associated molecular patterns (DAMPs). Danger signals are responsible for initiating or amplifying acute GVHD by

detect exogenous bacterial components, the host immune system identifies conserved structural moieties called pathogen-associated molecular patterns (PAMPs) that are found in microorganisms. Most innate immune cells express pattern recognition receptors (PRRs) and recognize PAMPs via PRRs, such as Toll-like receptors (TLRs) and the nucleotide-binding oligomerization domain (NOD)-like receptors (NLRs). The binding of PAMPs by PRRs on APCs activates the innate immune response, which induces the upregulation of cytokines and MHC class II costimulatory molecules and promotes DC migration to the T-cell area of lymph nodes. TLRs are transmembrane proteins located at the cell surface or in endosomes, while NLRs are located in the cytoplasm. To date, 11 TLRs have been identified in humans and 13 in the mouse [6].

Toll-like receptors (TLRs)

Ferrara et al. [7] clarified an essential role of TLR4 ligand and the lipopolysaccharide (LPS)/TLR4 pathway in the development of GVHD. Using mouse GVHD models, they showed that HCT recipients from an LPS-resistant donor led to significantly less GVHD compared with HCT recipients from an LPS-sensitive donor, and that an LPS antagonist reduces GVHD [8]. LPS was also shown to play a role in alloimmune lung injury. Garantziotis et al. [9] reported that LPS-induced lymphocytic lung inflammation was dependent on intact TLR4 signaling in donor-derived the enhancement of DC maturation and alloreactive T-cell responses. $L P S$ lipopolysaccharide, ATP adenosine triphosphate, $H M G B 1$ high mobility group box 1 protein, TLR Toll-like receptor, $N L R$ the nucleotide-binding oligomerization domain-like receptor, $P R R s$ pattern recognition receptors, $P 2 X_{7} R$ P2X purinoceptor 7 receptor, $R A G E$ receptor for advanced glycation endproducts, TCR T-cell receptor, $A P C$ antigen-presenting cell

hematopoietic cells. In the clinical setting, a trend toward a reduced incidence of severe acute GVHD was found when a TLR4 mutation associated with LPS hyporesponsiveness was present [10]. However, these associations were not statistically significant in recipients of HLA-matched sibling marrow transplants. Another study also failed to detect significant associations with polymorphisms of the genes encoding TLR4 and GVHD [11], although experimental murine GVHD models show the importance of the LPS/ TLR4 pathway in systemic and pulmonary GVHD. The different effects of TLR4 signaling in humans and mouse models might be caused in part by the bacterial gut decontamination performed routinely in clinical allogeneic HCT.

TLR7/8 recognizes single-stranded RNA and induces anti-viral response. Sykes et al. [12] showed that systemic exposure to the TLR7 agonist, R-848, is sufficient to permit access of activated T-cells to peripheral tissues and induce GVHD. Blazar et al. [13] administered the TLR7/8 agonist, 3M-011 after allogeneic HCT and observed increased GVHD mortality. Interestingly, the same group showed that mice injected with $3 \mathrm{M}-011$ before transplantation had reduced GVHD lethality. Ligation of TLR7/8 expressed primarily on APCs induced the expression of indoleamine 2,3-dioxygenase (IDO) which can suppress T-cell responses and promote tolerance and reduced injury in the colon $[14,15]$. These results suggest that certain TLRs can contribute to immune regulatory function. For instance, 
bacterial flagellin, a TLR5 agonist, regulates CD4 T-cell response by increasing the generation of regulatory T-cells (Tregs) [16] and protects epithelial cells from radiationinduced toxicity [17]. Hossain et al. [18] showed that pretransplant administration of fragellin reduced GVHD while preserving posttransplant donor immunity.

TLR9 recognizes cytosine-phosphorothioate-guanine oligodeoxynucleotides (CpG ODNs) that mimic bacterial and viral DNA and was also shown to be involved in GVHD $[19,20]$. TLR9 ${ }^{-/}$APCs have reduced allo-stimulatory activity and TLR9 ${ }^{-/-}$mice showed reduced gut GVHD morbidity and overall GVHD mortality [19, 20]. Ligation of TLR9 on host APCs with CpG ODNs enhanced donor T-cell responses, accelerating GVHD [13]. In the clinical setting, although the occurrence of acute GVHD was not different, TLR9 gene variants that are associated with reduced TLR9 expression were significantly associated with improved treatment-related mortality (TRM), overall survival (OS), and a lower relapse rate [21].

Nucleotide-binding oligomerization domain (NOD)like receptors (NLRs)

PAMPs are recognized not only by TLRs, but also by nucleotide-binding oligomerization domain (NOD)-like receptors (NLRs), which include proteins such as NACHT-, LRR-, and PYD-containing proteins (NALPs), NOD1, and NOD2. NLRs are involved in the secretion of inflammatory cytokines, such as interleukin-1 $\beta$ (IL-1 $\beta$ ) and IL-18. NOD2 recognizes muramyl dipeptide (MDP), a component of bacterial peptidoglycan, and induces $\mathrm{NF}-\kappa \mathrm{B}$ activation, leading to enhanced Th1 responses. Van den Brink et al. examined the role of NOD2 during GVHD. Unlike TLRs, they found that the use of NOD2 $2^{-1-}$ donor cells in wild-type recipients had no effect on GVHD [22]. Interestingly, they observed increased GVHD in NOD2 ${ }^{-/-}$HCT recipients and demonstrated that NOD2 deficiency in host hematopoietic cells exacerbates GVHD using chimeric mice. NOD2 $2^{-/-}$ DCs had a higher activation status and increased ability to induce T-cell proliferation during GVHD. These findings are in line with the observation that NOD2 $2^{-l-}$ DCs had enhanced ability to trigger inflammatory T-cell responses, and NOD2 $2^{-/-}$mice showed increased susceptibility to experimental colitis [23]. Watanabe et al. [23, 24] found that MDP activation of NOD2 regulates innate responses to intestinal microflora by downregulating multiple TLR responses and that the absence of such regulation leads to heightened Th1 responses.

In the clinical setting, several studies have shown that NOD2 single nucleotide polymorphisms (SNPs) are associated with GVHD [11, 25, 26]. Holler et al. [26] first reported an association between a greater incidence of GVHD and NOD2 SNPs of the donor or recipient. However, several

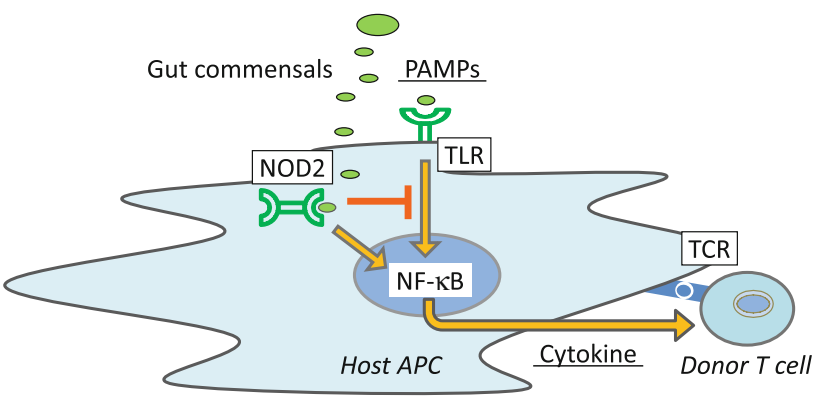

Fig. 2 PAMPs are recognized by NLRs and also by TLRs. A member of the nucleotide-binding oligomerization domain $(N O D)$ like receptors (TLRS), NOD2 recognizes pathogen-associated molecular patterns (PAMPs) as a primary sensor and induces NF-kB activation leading to enhance T-cell responses. In addition to inducing inflammatory T-cell responses, NOD2 functions negatively regulates TLR-mediated responses

other studies failed to confirm this association [27-31]. The conflicting results might be explained by multiple factors, including the NOD2 SNP frequency, overall incidence of GVHD, donor source, and intestinal microbial decontamination [32]. Moreover, NOD2 functions as a primary sensor of microbial products inducing inflammatory T-cell responses and also negatively regulates TLR-mediated responses (Fig. 2). This immunological balance might cause conflict in the association of NOD2 SNPs with GVHD.

\section{Endogenous danger signals: damage-associated molecular patterns (DAMPs)}

Endogenous noninfectious molecules, known as damageassociated molecular patterns (DAMPs), are released following conditioning regimen-induced tissue damage and play a critical role in GVHD (Table 1). Although the proinflammatory cytokines are not considered DAMPs, they serve as DAMPs, and the relationship between inflammatory cytokines and GVHD severity is well supported by animal models. The damaged, activated host tissues secrete cytokines, such as TNF- $\alpha$, IL-1, and IL-33. The consequences of the action of these cytokines are the increased expression of MHC antigens and adhesion molecules, which recruits effector cells and enhances the recognition of host alloantigens by donor T-cells. In addition to proinflammatory cytokines, DAMPs include intracellular molecules and extracellularly located ones. These are extracellular matrix fragments released by extracellular matrix degradation during tissue damage.

Adenosine triphosphate (ATP)

Zeiser et al. [33] demonstrated that extracellular adenosine triphosphate (ATP) released by dying cells serves as a 
Table 1 The role of DAMPs in the development of GVHD

\begin{tabular}{|c|c|c|c|c|}
\hline \multirow{3}{*}{$\frac{\text { DAMPs }}{\text { ATP }}$} & \multirow{3}{*}{$\begin{array}{l}\text { Receptors } \\
\text { NLRP3 }\left(\mathrm{P} 2 \mathrm{X}_{7} \mathrm{R}\right)\end{array}$} & \multicolumn{2}{|c|}{ Observations } & \multirow{2}{*}{$\frac{\text { References }}{[33]}$} \\
\hline & & Mouse & $\begin{array}{l}\text { Blockade of ATP-P2X } X_{7} \text { R signaling pathways decreased acute } \\
\text { GVHD }\end{array}$ & \\
\hline & & Human & $\begin{array}{l}\text { Polymorphisms of } \mathrm{P} 2 \mathrm{X}_{7} \mathrm{R}, \mathrm{NALP} 2 \text { and NALP3 are associated with } \\
\text { OS }\end{array}$ & {$[36,37]$} \\
\hline \multirow{2}{*}{$\begin{array}{r}\text { Heparan } \\
\text { sulfate }\end{array}$} & \multirow[t]{2}{*}{ TLR4 } & Mouse & $\alpha 1$-antitrypsin decreased serum heparan sulfate levels and GVHD & [39] \\
\hline & & Human & Serum heparan sulfate levels were associated with GVHD & {$[40]$} \\
\hline \multirow[t]{2}{*}{ HSPs } & \multirow[t]{2}{*}{$\begin{array}{l}\text { TLR2, TLR4, CD91, CD24, CD14 } \\
\text { and CD40 }\end{array}$} & Human & $\begin{array}{l}\text { HSP70 expression was correlated with high graft-versus-host } \\
\text { responses }\end{array}$ & [41] \\
\hline & & Human & Recipient HSP polymorphisms are associated with GVHD & {$[42]$} \\
\hline Uric acid & NLRP3 & Human & $\begin{array}{l}\text { Rasburicase reduced the serum uric acid levels and grade II-IV } \\
\text { GVHD }\end{array}$ & [44] \\
\hline Hyaluronan & TLR2, TLR4 and CD44 & Human & $\begin{array}{l}\text { CD44- hyaluronan contribute to lymphocytotropism to skin } \\
\text { GVHD }\end{array}$ & {$[45]$} \\
\hline S100 proteins & RAGE & Human & $\begin{array}{l}\text { S100 proteins were significantly more detected in saliva of GVHD } \\
\text { patients }\end{array}$ & [48] \\
\hline HMGB1 & $\begin{array}{l}\text { TLR2, TLR4, TLR9, RAGE and } \\
\text { CD24 }\end{array}$ & Human & $\begin{array}{l}\text { Polymorphisms of HMBG1 are associated with GVHD, TRM and } \\
\text { OS }\end{array}$ & {$[50]$} \\
\hline
\end{tabular}

$D A M P$ damage-associated molecular pattern, ATP adenosine triphosphate, $P 2 X 7 R$ P2X purinoceptor 7 receptor, NALP, NACHT, LRR, and $P Y D$ domains-containing protein, $T R M$ treatment-related mortality, $O S$ overall survival, $H S P$ heat shock protein, $H M G B 1$ high-mobility group box 1 , $R A G E$ receptor for advanced glycation end products

danger signal to enhance GVHD. ATP binds to P2X purinoceptor 7 receptor $\left(\mathrm{P} 2 \mathrm{X}_{7} \mathrm{R}\right)$ on host $\mathrm{APCs}$ and induces higher expression of the costimulatory molecules CD80 and CD86 on APCs. This receptor plays a central role in IL-1 $\beta$ secretion via NALP3 or the cryopyrin inflammasome, thereby also allowing more potent allo-stimulatory $\mathrm{T}$-cell priming. The pharmacological blockade of $\mathrm{P} 2 \mathrm{X}_{7} \mathrm{R}$ decreased the incidence of acute GVHD and increased the number of Tregs. They also showed that STAT5, which has several binding sites in the Foxp3 promoter region, was involved in Treg induction in $\mathrm{P} 2 \mathrm{X}_{7} \mathrm{R}$-deficient animals. CD39 dephosphorylates ATP to ADP and AMP and then CD73 dephosphorylates AMP to adenosine, which reduces inflammation. In the experimental GVHD models, the pharmacological blockade of CD73 enhanced GVHD activity [34], while an adenosine receptor agonist decreased acute GVHD [35]. In human recipients of allogeneic HCT, polymorphisms of $\mathrm{P} 2 \mathrm{X}_{7} \mathrm{R}, \mathrm{NALP} 2$, and NALP3 are associated with survival differences in allogeneic HCT patients [36, 37]. Therefore, $\mathrm{P} 2 \mathrm{X}_{7} \mathrm{R}$ signaling blockade might be a useful strategy for preventing acute GVHD caused by tissue damage during conditioning.

\section{Heparan sulfate (HS)}

Heparan sulfate (HS), an extracellular matrix component, can activate TLR4 on DC, which enhances DC maturation and alloreactive $\mathrm{T}$-cell responses [38]. Treatment with the serine protease inhibitor $\alpha 1$-antitrypsin (A1AT) decreased serum levels of HS, leading to a reduction in
GVHD severity. In the setting of allogeneic HCT, serum HS levels were increased and associated with the severity of GVHD. Tawara et al. [39] showed that A1AT treatment early after HCT reduced the expansion of alloreactive T-effector cells, but enhanced the recovery of Tregs and decreased mortality in experimental GVHD models. The administration of A1AT reduced serum proinflammatory cytokine levels and suppressed the LPSinduced secretion of proinflammatory cytokines in vitro, which enhanced the production of IL-10 in the host DCs. Another study showed that A1AT treatment reduces serum IL-32 levels and experimental GVHD severity [40]. These findings suggest that blocking HS release or administering A1AT might be an effective strategy for preventing GVHD.

Heat shock proteins (HSPs)

Heat shock proteins (HSPs) are ubiquitous chaperones that bind to and are involved in the folding and unfolding of other proteins. Extracellular HSPs released by dying cells activate innate immune responses via PRRs. HSPs also both induce the maturation of APCs and provide chaperoned polypeptides for triggering specific acquired immune responses. The 70 kilo Dalton HSP (HSP70) expression was correlated with high graft-versus-host responses in an in vitro-generated graft-versus-host reaction in human skin [41]. In human recipients of allogeneic HCT, recipient HSP polymorphisms are associated with a risk of acute GVHD [42]. 
Uric acid (UA)

Uric acid (UA) is released from dying cells and has adjuvant activity in vivo. UA enhanced DC maturation and amplified T-cell responses, and the elimination of UA in mouse models reduced the immune response [43]. Rasburicase is a recombinant urate-oxidase enzyme that catalyzes the oxidation of UA into an inactive soluble metabolite and is currently used to prevent tumor lysis syndrome. In a pilot trial, Brunner et al. [44] administered rasburicase to 23 patients beginning on the first day of conditioning therapy. They reported that rasburicase reduced the serum UA levels and there was significantly less grade II or higher acute GVHD in the rasburicase group compared with 44 comparable patients.

\section{Hyaluronan (HA)}

On tissue injury, high-molecular-weight (HMW) hyaluronan (HA), which is distributed ubiquitously in the extracellular matrix, is broken down into lower-molecular-weight (LMW) species. Milinkovic et al. [45] showed that hyaluronidase digestion of acute GVHD skin sections completely blocked CD44+ lymphocyte adherence to endothelium, suggesting that CD44- HA interactions contribute to lympho cytotropism to skin in acute GVHD. In addition to facilitating the recruitment of CD44+ leukocytes, LMW HA acts as an endogenous danger signal, leading to the activation of both innate and acquired immunity [46], although its relationship with GVHD is still underdetermined.

\section{S100 proteins}

S100 proteins are calcium binding and there are at least 21 different types of S100 protein. S100A8 and S100A9 are secreted by activated phagocytes and induce proinflammatory cytokines and adhesion molecules in endothelial cells [47]. Since a change in salivary constituents could reflect innate and adaptive immune responses during the development of GVHD, Chiusolo et al. [48] performed a proteome analysis of saliva from allogeneic HCT recipients with or without acute GVHD and healthy volunteers. They found significant differences among the three groups in terms of the frequency and levels of the proteins S100A8, S100A9, and S100A7, although further studies are needed to clarify the role of these proteins in the pathophysiology of acute GVHD.

\section{High mobility group box 1 protein (HMGB1)}

High mobility group box 1 protein (HMGB1) is expressed ubiquitously and located mostly in cell nuclei. HMGB1 is released on tissue damage as an endogenous DAMP and is actively produced by immune cells. Extracellular HMGB1 acts as a key molecule of innate immunity, downstream from persistent tissue injury, orchestrating inflammation, stem cell recruitment/activation, and eventual tissue remodeling [49]. Kornblit et al. [50] investigated HMGB1 polymorphisms and found associations between the HMGB1 genotype and outcome after allogeneic HCT following myeloablative (MA) conditioning, but not following nonmyeloablative (NMA) conditioning. The difference in the results between MA and NMA might be explained by a differential effect of HMGB1 depending on the intensity of the conditioning regimen. Inoue et al. [51] reported that a case of refractory acute GVHD complicated by thrombotic microangiopathy was treated successfully with recombinant thrombomodulin (rTM), which possesses the ability to neutralize HMGB1. TM is a membrane glycoprotein expressed mainly by vascular endothelial cells and is involved in coagulation and inflammation. Although the role of HMGB1 in GVHD is not completely clear, targeting innate immune cells and endothelial cells might lead to improved therapeutics in refractory acute GVHD complicated by thrombotic microangiopathy.

\section{Conclusions and further directions}

Despite improvements in clinical care, acute GVHD remains a major cause of morbidity and mortality for allogeneic HCT recipients and there is no standard treatment for patients with steroid-refractory GVHD. Most of the current prevention and treatment of acute GVHD targets donor T-cells. The blockade of DAMPs signaling involving ATP, HS, UA, and HMGB1 might be a useful strategy for preventing acute GVHD by reducing DC maturation and alloreactive T-cell responses. In addition to innate immune cells, endothelial cell dysfunction might lead to refractory GVHD treatment [52]. Luft et al. [52] revealed that rising levels of soluble TM, a marker of endothelial damage, were associated with steroid-refractory GVHD, suggesting that its pathogenesis involves progressive microangiopathy. Recently, DAMPs such as extracellular DNA, histones, and S100A8/A9 cause thrombotic microangiopathy [53]. An understanding of the role of PAMPs/DAMPs during the development of GVHD and also microangiopathies might offer new therapeutic approaches for steroid-refractory GVHD.

\section{References}

1. Barnes DW, Loutit JF, Micklem HS. "Secondary disease" of radiation chimeras: a syndrome due to lymphoid aplasia. Ann NY Acad Sci. 1962;99:374-85.

2. Billingham RE. The biology of graft-versus-host reactions. Harvey Lect. 1966;62:21-78. 
3. Shlomchik WD, Couzens MS, Tang CB, et al. Prevention of graft-versus-host disease by inactivation of host antigen-presenting cells. Science. 1999;285:412-5.

4. Duffner UA, Maeda Y, Cooke KR, et al. Host dendritic cells alone are sufficient to initiate acute graft-versus-host disease. J Immunol. 2004;172:7393-8.

5. Levine JE. Implications of TNF-alpha in the pathogenesis and management of GVHD. Int J Hematol. 2011;93:571-7.

6. Shin OS, Harris JB. Innate immunity and transplantation tolerance: the potential role of TLRs/NLRs in GVHD. Korean $\mathbf{J}$ Hematol. 2011;46:69-79.

7. Cooke KR, Hill GR, Crawford JM, et al. Tumor necrosis factoralpha production to lipopolysaccharide stimulation by donor cells predicts the severity of experimental acute graft-versus-host disease. J Clin Invest. 1998;102:1882-91.

8. Cooke KR, Gerbitz A, Crawford JM, et al. LPS antagonism reduces graft-versus-host disease and preserves graft-versus-leukemia activity after experimental bone marrow transplantation. J Clin Invest. 2001;107:1581-9.

9. Garantziotis S, Palmer SM, Snyder LD, et al. Alloimmune lung injury induced by local innate immune activation through inhaled lipopolysaccharide. Transplantation. 2007;84:1012-9.

10. Lorenz E, Schwartz DA, Martin PJ, et al. Association of TLR4 mutations and the risk for acute GVHD after HLA-matchedsibling hematopoietic stem cell transplantation. Biol Blood Marrow Transplant. 2001;7:384-7.

11. Elmaagacli AH, Koldehoff M, Hindahl H, et al. Mutations in innate immune system NOD2/CARD 15 and TLR-4 (Thr399Ile) genes influence the risk for severe acute graft-versus-host disease in patients who underwent an allogeneic transplantation. Transplantation. 2006;81:247-54.

12. Chakraverty R, Cote D, Buchli J, et al. An inflammatory checkpoint regulates recruitment of graft-versus-host reactive T-cells to peripheral tissues. J Exp Med. 2006;203:2021-31.

13. Taylor PA, Ehrhardt MJ, Lees CJ, et al. TLR agonists regulate alloresponses and uncover a critical role for donor APCs in allogeneic bone marrow rejection. Blood. 2008;112:3508-16.

14. Jasperson LK, Bucher C, Panoskaltsis-Mortari A, et al. Indoleamine 2,3-dioxygenase is a critical regulator of acute graft-versus-host disease lethality. Blood. 2008;111:3257-65.

15. Jasperson LK, Bucher C, Panoskaltsis-Mortari A, et al. Inducing the tryptophan catabolic pathway, indoleamine 2,3-dioxygenase (IDO), for suppression of graft-versus-host disease (GVHD) lethality. Blood. 2009;114:5062-70.

16. Crellin NK, Garcia RV, Hadisfar O, et al. Human CD4+ T-cells express TLR5 and its ligand flagellin enhances the suppressive capacity and expression of FOXP3 in CD4+ CD25+ T regulatory cells. J Immunol. 2005;175:8051-9.

17. Galkin VE, Yu X, Bielnicki J, et al. Divergence of quaternary structures among bacterial flagellar filaments. Science. 2008;320:382-5.

18. Hossain MS, Jaye DL, Pollack BP, et al. Flagellin, a TLR5 agonist, reduces graft-versus-host disease in allogeneic hematopoietic stem cell transplantation recipients while enhancing antiviral immunity. J Immunol. 2011;187:5130-40.

19. Calcaterra C, Sfondrini L, Rossini A, et al. Critical role of TLR9 in acute graft-versus-host disease. J Immunol. 2008;181:6132-9.

20. Heimesaat MM, Nogai A, Bereswill S, et al. MyD88/TLR9 mediated immunopathology and gut microbiota dynamics in a novel murine model of intestinal graft-versus-host disease. Gut. 2010;59:1079-87.

21. Elmaagacli AH, Koldehoff M, Beelen DW. Improved outcome of hematopoietic SCT in patients with homozygous gene variant of Toll-like receptor 9. Bone Marrow Transplant. 2009;44:295-302.

22. Penack O, Smith OM, Cunningham-Bussel A, et al. NOD2 regulates hematopoietic cell function during graft-versus-host disease. J Exp Med. 2009;206:2101-10.
23. Watanabe T, Asano N, Murray PJ, et al. Muramyl dipeptide activation of nucleotide-binding oligomerization domain 2 protects mice from experimental colitis. J Clin Invest. 2008;118: 545-59.

24. Watanabe T, Kitani A, Murray PJ, Strober W. NOD2 is a negative regulator of Toll-like receptor 2-mediated $\mathrm{T}$ helper type 1 responses. Nat Immunol. 2004;5:800-8.

25. Holler E, Rogler G, Herfarth $\mathrm{H}$, et al. Both donor and recipient NOD2/CARD15 mutations associate with transplant-related mortality and GvHD following allogeneic stem cell transplantation. Blood. 2004;104:889-94.

26. Holler E, Rogler G, Brenmoehl J, et al. Prognostic significance of NOD2/CARD15 variants in HLA-identical sibling hematopoietic stem cell transplantation: effect on long-term outcome is confirmed in 2 independent cohorts and may be modulated by the type of gastrointestinal decontamination. Blood. 2006;107:4189-93.

27. Granell M, Urbano-Ispizua A, Arostegui JI, et al. Effect of NOD2/CARD15 variants in T-cell depleted allogeneic stem cell transplantation. Haematologica. 2006;91:1372-6.

28. Mayor NP, Shaw BE, Hughes DA, et al. Single nucleotide polymorphisms in the NOD2/CARD15 gene are associated with an increased risk of relapse and death for patients with acute leukemia after hematopoietic stem-cell transplantation with unrelated donors. J Clin Oncol. 2007;25:4262-9.

29. Sairafi D, Uzunel M, Remberger M, et al. No impact of NOD2/ CARD15 on outcome after SCT. Bone Marrow Transplant. 2008;41:961-4.

30. Gruhn B, Intek J, Pfaffendorf N, et al. Polymorphism of interleukin-23 receptor gene but not of NOD2/CARD15 is associated with graft-versus-host disease after hematopoietic stem cell transplantation in children. Biol Blood Marrow Transplant. 2009;15:1571-7.

31. Tanabe T, Yamaguchi N, Matsuda K, et al. Association analysis of the NOD2 gene with susceptibility to graft-versus-host disease in a Japanese population. Int J Hematol. 2011;93:771-8.

32. Penack O, Holler E, van den Brink MR. Graft-versus-host disease: regulation by microbe-associated molecules and innate immune receptors. Blood. 2010;115:1865-72.

33. Wilhelm K, Ganesan J, Muller T, et al. Graft-versus-host disease is enhanced by extracellular ATP activating P2X7R. Nat Med. 2010;16:1434-8.

34. Tsukamoto $\mathrm{H}$, Chernogorova $\mathrm{P}$, Ayata $\mathrm{K}$, et al. Deficiency of CD73/ecto-5' ${ }^{\prime}$-nucleotidase in mice enhances acute graft-versushost disease. Blood. 2012;119:4554-64.

35. Lappas CM, Liu PC, Linden J, et al. Adenosine A2A receptor activation limits graft-versus-host disease after allogenic hematopoietic stem cell transplantation. J Leukoc Biol. 2010;87: 345-54.

36. Granell M, Urbano-Ispizua A, Pons A, et al. Common variants in NLRP2 and NLRP3 genes are strong prognostic factors for the outcome of HLA-identical sibling allogeneic stem cell transplantation. Blood. 2008;112:4337-42.

37. Lee KH, Park SS, Kim I, et al. P2X7 receptor polymorphism and clinical outcomes in HLA-matched sibling allogeneic hematopoietic stem cell transplantation. Haematologica. 2007;92:651-7.

38. Brennan TV, Lin L, Huang X, et al. Heparan sulfate, an endogenous TLR4 agonist, promotes acute GVHD after allogeneic stem cell transplantation. Blood. 2012;120:2899-908.

39. Tawara I, Sun Y, Lewis EC, et al. Alpha-1-antitrypsin monotherapy reduces graft-versus-host disease after experimental allogeneic bone marrow transplantation. Proc Natl Acad Sci USA. 2012;109:564-9.

40. Marcondes AM, Li X, Tabellini L, et al. Inhibition of IL-32 activation by alpha-1 antitrypsin suppresses alloreactivity and increases survival in an allogeneic murine marrow transplantation model. Blood. 2011;118:5031-9. 
41. Jarvis M, Marzolini M, Wang XN, et al. Heat shock protein 70 : correlation of expression with degree of graft-versus-host response and clinical graft-versus-host disease. Transplantation. 2003;76:849-53.

42. Bogunia-Kubik K, Lange A. HSP70-hom gene polymorphism in allogeneic hematopoietic stem-cell transplant recipients correlates with the development of acute graft-versus-host disease. Transplantation. 2005;79:815-20.

43. Shi Y, Evans JE, Rock KL. Molecular identification of a danger signal that alerts the immune system to dying cells. Nature. 2003;425:516-21.

44. Brunner AM, Thomas R. Spitzer TR, Chen Yi-Bin A et al. Urate Oxidase (Rasburicase) to Inhibit Graft Versus Host Disease (GVHD) After Myeloablative HLA-Matched Allogeneic Hematopoietic Cell Transplantation (HCT). ASH (Annual Meeting Abstracts) 2012;120: Abstract 3063.

45. Milinkovic M, Antin JH, Hergrueter CA, et al. CD44- hyaluronic acid interactions mediate shear-resistant binding of lymphocytes to dermal endothelium in acute cutaneous GVHD. Blood. 2004;103:740-2.

46. Shirali AC, Goldstein DR. Activation of the innate immune system by the endogenous ligand hyaluronan. Curr Opin Organ Transplant. 2008;13:20-5.

47. Ehrchen JM, Sunderkotter C, Foell D, et al. The endogenous Tolllike receptor 4 agonist S100A8/S100A9 (calprotectin) as innate amplifier of infection, autoimmunity, and cancer. J Leukoc Biol. 2009;86:557-66.

48. Chiusolo P, Giammarco S, Fanali C, et al. Salivary proteomic analysis and acute graft-versus-host disease after allogeneic hematopoietic stem cell transplantation. Biol Blood Marrow Transplant. 2013;19:888-92.

49. Castiglioni A, Canti V, Rovere-Querini P, Manfredi AA. Highmobility group box 1 (HMGB1) as a master regulator of innate immunity. Cell Tissue Res. 2011;343:189-99.

50. Kornblit B, Masmas T, Petersen SL, et al. Association of HMGB1 polymorphisms with outcome after allogeneic hematopoietic cell transplantation. Biol Blood Marrow Transplant. 2010;16:239-52.

51. Inoue Y, Kosugi S, Miura I, et al. Successful treatment of refractory acute GVHD complicated by severe intestinal transplant-associated thrombotic microangiopathy using recombinant thrombomodulin. Thromb Res. 2011;127:603-4.

52. Luft T, Dietrich S, Falk C, et al. Steroid-refractory GVHD: T-cell attack within a vulnerable endothelial system. Blood. 2011;118: 1685-92.

53. Fuchs TA, Kremer Hovinga JA, Schatzberg D et al. Circulating DNA and myeloperoxidase indicate disease activity in patients with thrombotic microangiopathies. Blood 2012;120:1157-1164. 